\title{
BMJ Open Protocol for a pilot randomised controlled trial of an educational programme for adults on chronic haemodialysis with fatigue (Fatigue- HD)
}

\author{
Janine F Farragher, ${ }^{1}$ Chandra Thomas, ${ }^{2}$ Pietro Ravani, ${ }^{2,3}$ Braden Manns, ${ }^{1,2}$ \\ Meghan J Elliott, ${ }^{2}$ Brenda R Hemmelgarn ${ }^{1,2}$
}

To cite: Farragher JF, Thomas C, Ravani P, et al. Protocol for a pilot randomised controlled trial of an educational programme for adults on chronic haemodialysis with fatigue (Fatigue-HD). BMJ Open 2019;9:e030333. doi:10.1136/ bmjopen-2019-030333

- Prepublication history for this paper is available online. To view these files, please visit the journal online (http://dx.doi. org/10.1136/bmjopen-2019030333).

Received 11 March 2019 Revised 01 July 2019 Accepted 09 July 2019

Check for updates

(c) Author(s) (or their employer(s)) 2019. Re-use permitted under CC BY-NC. No commercial re-use. See rights and permissions. Published by BMJ.

${ }^{1}$ Community Health Sciences, University of Calgary, Calgary, Alberta, Canada

${ }^{2}$ Medicine, University of Calgary, Calgary, Alberta, Canada ${ }^{3} 0$ 'Brien Institute of Public Health, University of Calgary, Calgary, Alberta, Canada

Correspondence to Dr Brenda R Hemmelgarn, University of Calgary, Calgary, Alberta, Canada;

Brenda.Hemmelgarn@ahs.ca

\section{ABSTRACT}

Introduction Fatigue is a pervasive symptom of endstage renal disease (ESRD) that is associated with low quality of life, disability and mortality, and has been identified as a top research priority by patients. We developed a personalised, web-supported educational programme (the Personal Energy Planning (PEP) programme) to teach people with ESRD to use energy management to manage fatigue. Preliminary studies have demonstrated positive effects on fatigue and life participation (ie, the ability to participate in valued day-today activities), which justifies the need for a randomised controlled trial (RCT) to better understand the efficacy of the programme. The objectives of the pilot RCT are to estimate RCT eligibility, recruitment and attrition rates, to inform the primary outcome measure and sample size for the RCT and to evaluate treatment fidelity among programme administrators.

Methods and analysis A parallel-arm, 1:1 pilot RCT will be conducted at four in-centre haemodialysis units in Calgary, Alberta, Canada. People on haemodialysis who report moderate or severe fatigue on the Fatigue Severity Scale, and meet other study eligibility criteria, will be invited to participate. Consenting participants will be randomised to undergo the 7-9 week 'PEP' programme or an active control, and followed for 12 weeks after the programme concludes. Information on eligibility, recruitment and attrition rates will be collected, and questionnaires assessing fatigue and life participation will be administered preintervention, midintervention, immediately postintervention and 12 weeks postintervention. Analyses will include calculation of eligibility, recruitment and attrition rates; power considerations for the full-scale RCT and evaluation of treatment fidelity of programme administrators.

Ethics and dissemination Risks associated with this study are minor. Patients may experience emotional discomfort while filling out study questionnaires. They will be advised to skip any questions that make them uncomfortable. Potential benefits of participating include any benefit derived from the study intervention, and contributing to research that may benefit people with kidney disease in the future. Trial results will be disseminated via publication in an academic journal and
Strengths and limitations of this study

- The pilot randomised controlled trial protocol was developed in accordance with the Standard Protocol Items: Recommendations for Interventional Trials (SPIRIT) guidelines.

- Use of an extensive standardised training protocol for the study intervention to maximise treatment fidelity across programme administrators.

- Use of an active control condition and blinding of patients and outcome assessors to treatment allocation status, to control for the placebo effect.

- Exclusion of non-English-speaking patients limits its generalisability to non-English end-stage renal disease populations.

presentation at academic conferences. The study has been approved by the Conjoint Health Research Ethics Board at the University of Calgary (ID \#18-1657).

\section{INTRODUCTION}

Fatigue is a pervasive symptom of end-stage renal disease (ESRD) experienced by an estimated 7 in 10 people on maintenance dialysis therapy. ${ }^{1}$ Fatigue has been defined as an 'unusual, excessive or whole body tiredness, disproportionate or unrelated to activity or exertion', 2 and is associated with a variety of adverse clinical outcomes, including low quality of life, ${ }^{34}$ hospitalisations ${ }^{56}$ and mortality. ${ }^{7}$ Fatigue is viewed as a complex, biopsychosocial symptom of illness, ${ }^{89}$ which can be affected by biological, psychological, behavioural and treatment-related factors in ESRD. ${ }^{10}$ Biological factors believed to trigger and perpetuate fatigue in ESRD include anaemia, inflammation and uraemia, ${ }^{10}$ while treatment-related factors, such as postdialysis malaise, dialysis adequacy and dialysis modality, have also been linked to 
patient fatigue. ${ }^{10}$ Psychologically, negative thoughts and beliefs about ESRD and fatigue are purported to result in maladaptive coping responses to fatigue,${ }^{11}$ which can worsen the experience of fatigue and might increase the risk of depression and anxiety. ${ }^{12}$ Patient behaviours, such as physical activity levels, sleep patterns, all-or-nothing responses to fatigue and avoidance of activity, are also associated with fatigue in ESRD. ${ }^{10}{ }^{13}$ Patients with ESRD have identified fatigue and its negative impact on their life participation (ie, ability to participate in valued day-to-day activities) as top priorities for research, ${ }^{5} 14$ justifying the need to explore interventions that can reduce fatigue and maximise life participation in this patient population.

The most well-researched approaches for managing fatigue in ESRD are erythropoietin therapy to target anaemia ${ }^{15}$ and exercise training to increase physical fitness. ${ }^{16}$ These approaches, while efficacious for some patients, also have limitations. For example, erythropoietin therapy does not address the multiple fatigue mechanisms in ESRD beyond anaemia, while exercise training has been challenging to implement and sustain in ESRD clinical practice, due to factors such as insufficient staff expertise and low patient motivation. ${ }^{17}$ Cognitive behavioural therapy for fatigue is an approach targeting unhelpful beliefs and behaviours related to fatigue that has shown promising results in other populations, ${ }^{1819}$ and is currently under investigation for people with ESRD. ${ }^{11}$ Energy management education (EME) is yet another approach to fatigue management that has also been associated with improvements in other chronic disease populations, such as multiple sclerosis ${ }^{20-22}$ and cardiac disease. ${ }^{2324}$ The theory behind EME is that fatigue in chronic disease is exacerbated when an individual's energy capacity exceeds their energy expenditure during day-to-day activities, which can consequently interfere with life participation. The objective of EME is, therefore, to provide practical strategies (eg, prioritising, using efficient body postures and organising the home environment) to reduce energy expenditure during everyday life, minimise fatigue and maximise life participation. EME may be well-suited to meet the needs of people with ESRD, as they have been found to have a reduced energy capacity compared with healthy populations, ${ }^{6}$ and must also expend extra energy on multiple health management tasks associated with dialysis (eg, planning and preparing renal-friendly meals, attending dialysis or performing home dialysis, and monitoring fluid intake and blood pressure) in addition to usual daily activities. To date, EME has never been studied in the ESRD population.

We developed a personalised, web-supported EME programme (the 'Personal Energy Planning (PEP)' programme) that has been tailored for the ESRD population in several ways. The programme is designed specifically to target the impact of fatigue on life participation, in accordance with patient-identified priorities, using a personalised, goal-focused intervention approach. It is also delivered in a concise, flexible and web-supported format with minimal homework, to accommodate patients' time restrictions resulting from their dialysis schedules. Preliminary acceptability testing found that the programme was both practical and well-received based on feedback from patient interviews, ${ }^{25}$ while five single-case studies revealed small-to-moderate improvements in fatigue and life participation associated with the programme in people with ESRD (according to Tau-U statistic of effect-size estimates and in-depth patient interviews). ${ }^{25}$ These positive preliminary findings justify a randomised controlled trial (RCT) to more conclusively establish the efficacy of the PEP programme.

However, additional information is first needed to design and plan an RCT. First, we need to establish the feasibility of an RCT on the PEP programme. Poor recruitment and high attrition rates are common in clinical trials involving ESRD patients, with high illness burden as one possible factor. This could be problematic for a study of an educational programme, such as the PEP programme, that will require substantial patient engagement and participation. Second, we need to understand the feasibility of training non-rehabilitation clinicians (eg, nurses) to administer the PEP programme for future knowledge translation and programme planning purposes, as rehabilitation therapists (who typically administer EME programme) are often absent from dialysis units. Finally, we need to collect more data on the effects of the programme on possible primary outcomes (fatigue and life participation) to determine the optimal primary outcome measure for an RCT, to estimate the sample size for an RCT and to establish longer-term effects of the PEP programme on patient fatigue and life participation.

\section{OBJECTIVES}

\section{Primary objective}

1. To estimate the proportion of ESRD patients that are eligible for an RCT of the PEP programme, will consent to participate and will complete all study procedures.

\section{Secondary objectives}

1. To identify the fatigue or life participation outcome measure, which is most sensitive to change related to the intervention, and to estimate the treatment effect size and variability for RCT sample size calculations.

2. To explore the effects of the PEP programme on fatigue and life participation at 3 months post-treatment.

3. To examine treatment fidelity to the PEP programme among non-rehabilitation clinical staff after participating in a short programme training course.

\section{METHODS \\ Trial design}

Parallel group, 1:1, pilot RCT

\section{Participant identification}

Participants will be recruited from four in-centre haemodialysis units in Calgary, Alberta, Canada. Patients who 
Table 1 Study eligibility criteria

\begin{tabular}{|c|c|}
\hline Inclusion criteria & Exclusion criteria \\
\hline $\begin{array}{l}\text { 1. Aged } \geq 18 \text { years } \\
\text { 2. On chronic dialysis therapy for } \geq 3 \text { months at the time of } \\
\text { recruitment } \\
\text { 3. Clinically and cognitively stable (able to provide informed } \\
\text { consent) } \\
\text { 4. Scores an average of } \geq 4 \text { on items } 5,7,8 \text { and } 9 \text { of the } \\
\text { Fatigue Severity Scale }\end{array}$ & $\begin{array}{l}\text { 1. Inadequate written and verbal English comprehension for } \\
\text { study activities } \\
\text { 2. Plan in place to discontinue in-centre haemodialysis } \\
\text { at participating centre within } 6 \text { months of the time } \\
\text { of recruitment (due to modality change, relocation, } \\
\text { transplantation or dialysis withdrawal) } \\
\text { 3. Resides in a nursing home facility } \\
\text { 4. Preclusive visual impairment }\end{array}$ \\
\hline
\end{tabular}

would be potentially eligible and interested in the study will be identified by clinical staff and approached to assess their interest in the study. Interested patients will undergo a comprehensive informed consent process. Written informed consent will be obtained before any study procedures are undertaken. Consenting participants will undergo full eligibility screening, using the study eligibility criteria (table 1). Items 5, 7, 8 and 9 of the Fatigue Severity Scale (FSS) are being used to identify eligible patients because these items specifically ask about the impact of fatigue on life participation, which is the intended focus of the intervention. Consenting and eligible patients will be invited to participate in the study.

\section{Randomisation and concealment}

Participants will be allocated equally (1:1) to intervention or control. Permuted blocked randomisation with randomly varied block sizes of 2-4 will be performed, and randomisation will be stratified by dialysis unit. Participants will be allocated using a computer-generated random number sequence. Randomisation will be performed by a research team member who is not involved in other aspects of the study, to maintain allocation concealment.

\section{Blinding}

Study participants will be blinded as to which treatment condition is the true treatment under study (intervention or active control). All patient study materials and communications will be left vague, describing the study purpose as being an investigation of an 'educational programme' for adults with fatigue. Blinding of treatment administrators will not be feasible, given their required level of familiarity with both the treatment and control conditions.

\section{Treatment: the 'PEP' programme}

Participants randomised to the treatment condition will undergo the 'PEP' programme. The PEP programme is a two-part EME programme that provides general education about energy management and individualised training to develop personalised energy management strategies. The programme is delivered over 7-9 weekly sessions, dependent on individual patient needs and rates of progress. Sessions are $20-30$ min in duration each, and administered either in person or via telephone (based on patient preference). The programme is administered by a trained study clinician (occupational therapist or nurse).

Part 1: Participants complete two educational computer modules (20-30 min each) that explain basic principles related to energy management (eg, energy budgeting, prioritising and seven key energy-saving strategies), and include activities and exercises to reinforce key concepts. The modules are publicly accessible online (www. pepmodule1.com and www.pepmodule2.com), and can be completed by patients independently (with support provided to access technology, as needed).

Part 2: Participants learn how to apply the energy management principles from Part 1 to accomplish their own life participation goals. First, participants work with a study clinician to identify three personal life participation goals (eg, to be able to do the grocery shopping weekly). They then complete a web module (www.pepmodule3. com) that explains a method to identify personalised energy management strategies that will facilitate their goals. The method is an adapted version of the Cognitive Orientation to Occupational Performance (CO-OP) intervention, ${ }^{26}$ which is an evidence-based approach to problem-solving and skill acquisition. ${ }^{27}$ Key elements of CO-OP used in PEP include dynamic performance analysis (ie, analysing where the participant is expending excessive amounts of energy during each goal activity), goal-plan-do-check (ie, generating energy management 'plans', 'doing' the plans and 'checking' to see if they work) and guided discovery (a method of questioning and cueing used by the study clinician to enable the participant to discover energy management strategies themselves). Participants spend 5-7 programme sessions (15-30 min each) applying the CO-OP approach with the study clinician to develop and test personalised energy management strategies for accomplishing their goals. The process is continued until an optimal performance solution is found for each goal, or the programme maximum of 9 weekly treatment sessions is reached (whichever comes first). Participants are also given a programme workbook to guide them throughout the PEP programme.

\section{Control: general information about kidney disease}

Participants randomised to the control condition will review information from the Kidney School learning modules, ${ }^{28}$ during $6-8$ individual sessions with a trained 
study clinician (occupational therapist or nurse). The modules contain general information about managing kidney disease, addressing topics, such as diet and heart health. Sessions will take place either in person or via telephone (based on patient preference). Use of this active control condition will minimise the risk of bias associated with patients receiving extra staff attention during the treatment condition.

\section{Treatment adherence}

Study coordinators will monitor and encourage participant adherence to the treatment protocol during weekly visits. All missed or incomplete treatment sessions will be documented.

\section{Staff training}

Treatment administrators will undergo training in the treatment and control protocols, and each will be responsible for providing both treatments. Training for the treatment protocol will consist of three 90 min sessions, while control protocol training will include one $60 \mathrm{~min}$ session. A training manual for treatment and control conditions will also be provided to support the administrators. Training materials can be obtained by contacting the study corresponding author.

\section{Concomitant care}

Patients enrolled in the trial will continue to receive and undergo all usual clinical care activities. Changes in clinical care or status during the study that could influence outcomes of fatigue and life participation (eg, exercise regimens and haemoglobin level changes) will be documented.

\section{Data collection}

Demographic and clinical data (table 2) will be collected for each consenting participant at the time of their first study visit by a trained study assessor, either through chart review or participant interview.

The number of screened patients who meet study inclusion and exclusion criteria, consent to participation and randomisation and complete all study procedures will be documented by study staff. Follow-up information (including recent hospitalisations, illnesses,

\begin{tabular}{ll}
\hline Table 2 Demographic and clinical study variables \\
\hline Demographic & Clinical \\
\hline Age & Dialysis vintage \\
Sex & Comorbidities \\
Residence type & Most recent haemoglobin \\
\hline Living status & Most recent albumin \\
Marital status & $\begin{array}{l}\text { Activities of daily living } \\
\text { independence }\end{array}$ \\
Employment & Cognitive function (Mini- \\
& COG) \\
\hline Education & Depression (PHQ-2) \\
\hline
\end{tabular}

dialysis changes, exercise changes, serum haemoglobin and albumin) will be documented at each follow-up visit.

The following questionnaires will be used to measure fatigue and life participation outcomes. These questionnaires were selected based on patient-reported priorities, such as minimising the burden of administration, limiting the recall period and capturing the impact of fatigue on life participation. ${ }^{5}$

\section{Fatigue Severity Scale}

The $\mathrm{FSS}^{29}$ asks individuals to rate, on a Likert scale from 1 to 7 , the severity of their fatigue and its impact on their life during the past week. The FSS is a valid, reliable and responsive measure ${ }^{30} 31$ that has previously been used in the dialysis population. ${ }^{12}$

\section{Fatigue Management Questionnaire}

The Fatigue Management Questionnaire asks participants to rate various aspects of their fatigue management (eg, competence, satisfaction and self-efficacy) on a Likert Scale of 1-10. The questionnaire was created for this study to fill a gap in assessments that measure life participation and self-efficacy specifically related to fatigue management.

\section{Modified Fatigue Impact Scale}

The Modified Fatigue Impact Scale ${ }^{32}$ is a 21-item Likertbased scale that assesses the effects of fatigue on physical, cognitive and psychosocial functioning. The Fatigue Impact Scale has frequently been used as an outcome measure in EME studies.

\section{Reintegration to Normal Living Index}

The Reintegration to Normal Living Index (RNLI) ${ }^{33}$ assesses the degree to which individuals who have experienced traumatic or incapacitating illness achieve reintegration into normal social activities, using 11 declarative statements that are accompanied by a visual analogue scale. The RNLI has been found to have strong validity and reliability in multiple disease populations. ${ }^{34}$

\section{Canadian Occupational Performance Measure}

The Canadian Occupational Performance Measure $(\mathrm{COPM})^{35}$ is designed to capture a client's perception of his/her performance in three priority tasks of everyday living. It asks individuals to rate, on a 10-point Likert scale, the importance of three self-chosen priority activities, their current perceived performance on the priority activities and their satisfaction with that performance. The COPM has been found to be a valid, reliable, clinically useful and responsive outcome measure in multiple disease populations. ${ }^{36}$

The fatigue and life participation questionnaires will be administered at four time points (figure 1) (except the COPM, which will not be administered at baseline).

1. Preintervention baseline.

2. Post-Part 1 of the PEP programme (just prior to commencing Part 2, session 1). 


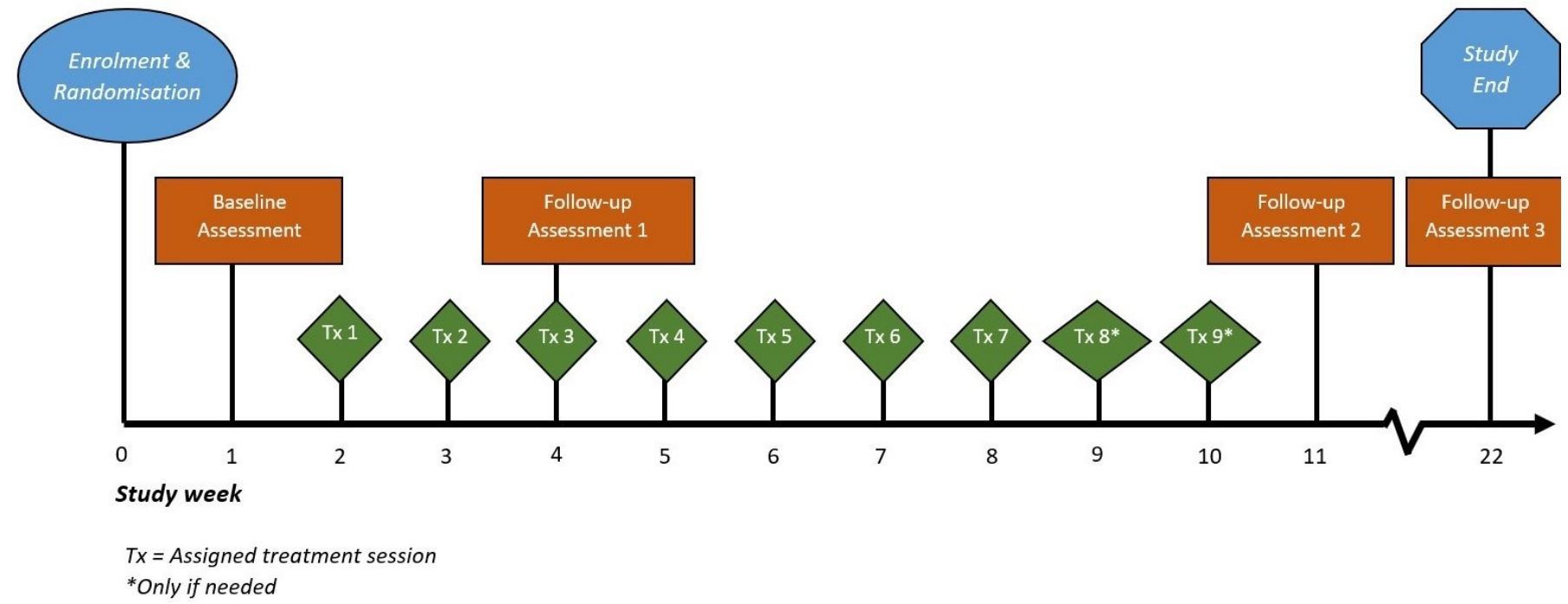

Figure 1 Participant timeline. *Only if needed. Tx, assigned treatment session.

3. Post-Part 2 of the PEP programme (1 week after the final study visit).

4. Twelve weeks after the final study visit.

Questionnaires will be completed before, during or after a dialysis session, according to participant preference. The timing and location of questionnaire completion will be kept consistent across assessment time points for each participant.

All treatment sessions (excluding computer modules) will be audio-recorded on an audio-recording device. Two sessions per participant randomised to the treatment condition will then be randomly selected and used to evaluate treatment fidelity of the programme administrators, according to the CO-OP fidelity checklist. The checklist includes 26 items, each scored on a scale of $0-5$, that measure the extent of use of various key elements of the treatment approach by the treatment administrator.

\section{Data management and confidentiality}

Study data will be recorded onto standardised paper study forms at the time of collection. Data will be anonymised by assigning each participant an unidentifiable study ID number at the time of enrolment, which will be used to identify them for all study materials. Paper data forms will immediately be filed and stored in a locked office area, and signed study consent forms will be filed and stored separately from data forms to maintain participant anonymity.

Study data will subsequently be entered into a secure database by a research assistant. The database will be password-protected and stored on a secure server, with access restricted to authorised users of the server. Range checks for data values will be performed after data entry, to promote data quality.

Audio recordings of study sessions will also be transferred onto a secure server, and deleted from their original recording device at the time of transfer. A sample of the audio recordings will subsequently be transcribed into text by the team transcriptionist and stored on the secure server. Data files and documents will be destroyed 7 years after the project is closed.

\section{Protocol deviations and amendments}

Protocol deviations are reported in box 1 . Any mid-study protocol modifications will be submitted to co-investigators and Research Ethics Board for approval and communicated to study participants and the trial registry once approved.

\section{Missed study treatment or assessment appointment}

Missed study sessions will be addressed as outlined in table 3. The study treatment and the assessment schedule has been designed with the flexibility to accommodate the frequent changes in health status and fatigue levels experienced by this population, which may cause occasional missed study appointments.

\section{Data analysis}

Demographic and clinical data will be reported as means and SDs for continuous parametric data, medians and

\section{Box 1. Protocol deviations}

a. Failure to initiate treatment within 2 weeks of study screening and enrolment.

b. Missed $\geq 3$ consecutive treatment or control sessions, leading to discontinuation of assigned treatment condition (but not withdrawal from the study).

c. Missed $\geq 2$ consecutive study assessment visits, leading to non-completion of an assessment package (but not withdrawal from the study).

d. Participants switch end-stage renal disease treatment modality during the course of the study.

e. Participants are hospitalised overnight during the course of the study.

f. Dropouts and their causes (eg, withdrawal of consent* or transfer to another centre). 
Table 3 Protocol for missed study sessions

\begin{tabular}{|c|c|}
\hline Missed session details & Response \\
\hline $\begin{array}{l}\text { Participant misses one to two consecutive weekly treatment } \\
\text { sessions }\end{array}$ & $\begin{array}{l}\text { Missed appointment(s) will be documented } \\
\text { The scheduled treatment session will be delayed until the } \\
\text { next weekly session } \\
\text { Dates of remaining assessment and treatment sessions will } \\
\text { be delayed accordingly }\end{array}$ \\
\hline $\begin{array}{l}\text { Participant misses three or more consecutive weekly } \\
\text { treatment sessions }\end{array}$ & $\begin{array}{l}\text { Missed appointments will be documented } \\
\text { Treatment protocol will be discontinued } \\
\text { Treatment discontinuation will be recorded as a protocol } \\
\text { deviation } \\
\text { Assessment schedule will carry on as planned, regardless of } \\
\text { missed treatment sessions }\end{array}$ \\
\hline $\begin{array}{l}\text { Participant misses scheduled assessment appointment date } \\
\text { and does not complete it during the week of the scheduled } \\
\text { date, but completes it the following week }\end{array}$ & $\begin{array}{l}\text { Missed appointment date will be documented } \\
\text { The scheduled assessment will be delayed to the following } \\
\text { week } \\
\text { Dates of remaining treatment and assessment sessions will } \\
\text { be delayed accordingly }\end{array}$ \\
\hline
\end{tabular}

ranges for continuous non-parametric data, and frequencies and percentages for categorical data.

The proportion of patients meeting each of the feasibility endpoints (eligibility, recruitment and attrition rates), with accompanying $95 \%$ CIs, will be calculated.

Assuming a normal distribution, standardised effect sizes for each fatigue and disability outcome measure will be calculated for both immediate postintervention and 3 months postintervention, as follows:

Cohen's $D=\frac{\text { Mean pre-post change }(\text { treatment })-\text { Mean pre-post change (control) }}{\text { Standard deviation (pooled) }}$

These data will be analysed using intention-to-treat analysis. Sample size calculations for the RCT will be made using the treatment effect size and variance estimates from the immediate postintervention change data for the selected outcome measure. Missing follow-up data will be addressed using pairwise deletion.

The treatment fidelity of treatment administrators will be analysed by calculating an average score out of 5 on the CO-OP fidelity checklist, for one treatment session per participant randomised to the treatment condition.

\section{SAMPLE SIZE AND FEASIBILITY}

A sample size of 40 patients (20 per treatment arm) was chosen based on the recommendations of Whitehead $e t$ $a l .{ }^{37}$ They suggest that this sample size will provide a sufficiently precise estimate of the treatment effect to minimise the sample needed for a future RCT, assuming $80 \%$ power, a small-medium effect size (which is expected based on our preliminary data ${ }^{25}$ ) and an attrition rate of no more than $\leq 25 \%$.

There are approximately 425 prevalent patients on haemodialysis in total at the four participating clinical sites. We project that approximately half (212 patients) will be identified as potential participants with fatigue, based on preliminary symptom screening data from the sites. Given that this is a high-priority research area among dialysis patients, we conservatively estimate that at least $25 \%$ (56 patients) of patients with fatigue will agree to participate. Furthermore, we expect no more than $25 \%$ of patients will subsequently be excluded during eligibility screening. This will enable us to achieve the target sample size of 40 patients.

\section{Patient and public involvement}

Patients have been involved, both directly and indirectly, in multiple aspects of this research project. The intervention under study was developed in response to the results of patient engagement research, which identified a need to further investigate fatigue management in renal disease. ${ }^{14}$ Two patients were involved as key informants in the development of the intervention under study, providing consultation and feedback on the first intervention prototype through a series of individual interviews that led to several programme modifications (eg, clarification of key content and simplification of design features). Two patients were also consulted about the control condition to be used in this study, and their feedback led to modifications, such as individualisation of the content material for specific patient interests and needs. Our current study team includes a patient partner who will be consulted about patient-related issues that arise during the study, the interpretation of results and strategies to optimise dissemination and uptake. 


\section{ETHICAL CONSIDERATIONS}

\section{Risks and benefits}

As part of their baseline assessment, participants will complete the PHQ-2 depression screening assessment. This assessment may identify individuals who have, or are at risk for, clinical depression. Any individual who scores $>2$ on the PHQ-2 will be offered connexion to support services, such as referral to their clinical social worker or to a local counselling centre. Study participants will also have to complete several study questionnaires, and participate in the PEP programme treatment sessions. There is a risk that patients may experience short-term fatigue, or, uncomfortable or unpleasant emotions in response to some of the questions in the study questionnaires. Participants will, therefore, be advised that they can skip any questions or study procedures that make them uncomfortable.

Direct benefits of participating are those which may be gained from completing the study intervention, such as improved fatigue management, improved knowledge about kidney disease and/or an increased staff attention. Indirect benefits include the potential that others with kidney disease may benefit from the study findings in the future.

\section{Data Safety and Monitoring Board}

As the proposed study is small and its risks to participants are low, a Data and Safety Monitoring Board is not needed. Monitoring for potential risks (eg, fatigue and discomfort) will be performed by those interacting directly with the patient during the study (the study clinicians and assessor). If any unexpected concerns arise that cannot be immediately mitigated, the concerns will be brought forth to the principal investigators for further discussion and decision-making.

\section{Dissemination plan}

Trial results will be disseminated to patients with a summary sheet that will outline the trial findings in lay language. Results will be disseminated to healthcare professionals and researchers via publication in an academic journal and presentation at academic conferences.

\section{DISCUSSION}

Fatigue is a common and disabling symptom of ESRD ${ }^{1-6}$ that has traditionally been challenging to mitigate due to its complex and non-specific aetiology. Results from patient-reported outcome and engagement studies have highlighted the need to continue to explore new fatigue management interventions for people with ESRD. ${ }^{14}$ EME is an approach that has been associated with positive fatigue-related outcomes in other chronic disease populations. For example, in people with Multiple Sclerosis, RCTs have found that EME reduces patient fatigue and its impact on physical, cognitive and psychosocial functioning, and improves self-efficacy. ${ }^{20-22} 3839$ Earlier-phase studies in acquired brain injury, ${ }^{40}$ cardiac disease ${ }^{23} 24$ and post-polio ${ }^{41}$ have similarly shown positive effects on fatigue and other related, high-priority outcomes, such as life participation. ${ }^{23}{ }^{40}$ Furthermore, single-case studies conducted in a small sample of ESRD patients have generated promising findings regarding the effects of the PEP programme on fatigue and life participation in people on chronic dialysis, ${ }^{25}$ suggesting that this approach has potential to fill an important gap in ESRD care. However, studies in ESRD have, thus, far lacked important design elements, such as blinding, randomisation and sample representativeness, leaving the true potential of the PEP programme unclear.

This proposed pilot RCT will provide several pieces of feasibility information to help to plan an RCT that can more conclusively establish the efficacy of the PEP programme in people with ESRD. It will provide more accurate preliminary estimates of programme effect sizes that are currently available, enabling greater precision in RCT power and sample size calculations. It will also provide estimates of eligibility, recruitment and attrition rates, which will help to ensure that adequate numbers of patients are approached for the RCT. Finally, it will help us to maximise fidelity to the treatment protocol in the RCT by providing information on the effectiveness of the current staff training programme, and the potential need to involve rehabilitation specialists in future programme research and implementation. These will all be necessary factors to ensure successful future implementation of an RCT.

The proposed pilot RCT has a number of strengths. The programme under investigation (the PEP programme) has been tailored specifically to meet the needs of the ESRD population: it is designed to facilitate participation in meaningful activities, which is a high priority for ESRD patients, and is delivered in a flexible format to accommodate the dialysis schedule. Patients have also been consulted and provided input at several stages of intervention development and testing. The study protocol was developed using the SPIRIT guidelines for a pilot RCT protocol, increasing the likelihood that important study design elements have been addressed. We have also developed a standardised training and administration protocol for the PEP programme, which we anticipate will maximise treatment fidelity and consistency across programme administrators. An active control condition to blind patients to their treatment allocation status will further increase the confidence in our study findings, by controlling for the placebo effect.

Our study also has limitations. First, we are excluding non-English-speaking patients from the study, which limits its generalisability to non-English-speaking ESRD populations. However, the findings from this study may help to justify developing programme materials in alternative languages that are accessible to a wider range of renal patients. We are also excluding patients outside of the in-centre haemodialysis population who also experience a high burden of fatigue (eg, predialysis patients, peritoneal dialysis patients and home haemodialysis patients). This study should be viewed as an important first step in establishing the potential for the PEP programme that 
can lay the groundwork for future research into EME in other renal populations. Finally, we are unable to blind treatment administrators to treatment allocation, due to our inability to conceal which study condition is the treatment condition. We perceive blinding to be unfeasible because treatment administrators would be able to identify the treatment condition, based on inequities between the two conditions in the amount of content dedicated to fatigue and the length of time spent on staff training. The infeasibility of blinding is a well-recognised limitation of trials studying psychosocial or behavioural interventions that are not easily matched with an equivalent control.

In conclusion, the findings from this pilot RCT will further our understanding of a programme that has potential to address the challenging problem of fatigue in the ESRD patient population.

\section{Trial status}

The study started recruitment at the end of February 2019. Recruitment will continue until August 2019. Data collection will conclude in January 2020.

Contributors JF and BH led the design and writing of the pilot RCT protocol. CT and BM helped with the development of the participant identification plan, and provided advice on other key study issues. PR and ME contributed feedback on trial design. All the authors contributed important intellectual content to the written protocol and approved the final version for publication.

Funding This research is funded through the Interdisciplinary Chronic Disease Collaboration and the Roy and Vi Baay Chair in Kidney Research.

Competing interests None declared.

Patient consent for publication Not required.

Ethics approval Ethics approval for the study has been obtained from the Conjoint Health Research Ethics Board (CHREB) at the University of Calgary.

Provenance and peer review Not commissioned; externally peer reviewed.

Open access This is an open access article distributed in accordance with the Creative Commons Attribution Non Commercial (CC BY-NC 4.0) license, which permits others to distribute, remix, adapt, build upon this work non-commercially, and license their derivative works on different terms, provided the original work is properly cited, appropriate credit is given, any changes made indicated, and the use is non-commercial. See: http://creativecommons.org/licenses/by-nc/4.0/.

\section{REFERENCES}

1. Murtagh FEM, Addington-Hall J, Higginson IJ. The prevalence of symptoms in end-stage renal disease: a systematic review. Adv Chronic Kidney Dis 2007;14:82-99.

2. Piper BF. Fatigue and cancer: inevitable companions? Support Care Cancer 1993;1:285-6.

3. Weisbord SD, Fried LF, Mor MK, et al. Renal provider recognition of symptoms in patients on maintenance hemodialysis. CJASN 2007;2:960-7.

4. Davison SN, Jhangri GS. Impact of Pain and Symptom Burden on the Health-Related Quality of Life of Hemodialysis Patients. J Pain Symptom Manage 2010;39:477-85.

5. Ju A, Unruh M, Davison S, et al. Establishing a core outcome measure for fatigue in patients on hemodialysis: a standardized outcomes in Nephrology-Hemodialysis (SONG-HD) consensus workshop report. American Journal of Kidney Diseases 2018;72:104-12.

6. Heiwe S, Clyne N, Dahlgren MA. Living with chronic renal failure: patients' experiences of their physical and functional capacity. Physiother. Res. Int. 2003;8:167-77.

7. Bossola M, Di Stasio E, Antocicco M, et al. Fatigue is associated with increased risk of mortality in patients on chronic hemodialysis. Nephron 2015;130:113-8.

8. Ormstad H, Eilertsen $\mathrm{G}$. A biopsychosocial model of fatigue and depression following stroke. Med Hypotheses 2015;85:835-41.
9. Hwang SS, Chang VT, Rue M, et al. Multidimensional independent predictors of cancer-related fatigue. J Pain Symptom Manage 2003;26:604-14.

10. Artom M, Moss-Morris R, Caskey F, et al. Fatigue in advanced kidney disease. Kidney Int 2014;86:497-505.

11. Cognitive-behavioural therapy (CBT) for renal fatigue (BReF): a feasibility randomised-controlled trial of CBT for the management of fatigue in hae. - PubMed - NCBI [Internet. Available: https://www. ncbi.nlm.nih.gov/pubmed/29523571 [Accessed cited 2019 Jun 20].

12. Farragher JF, Polatajko HJ, Jassal SV. The relationship between fatigue and depression in adults with end-stage renal disease on chronic in-hospital hemodialysis: a scoping review. J Pain Symptom Manage 2017;53:783-803.

13. Jhamb M, Weisbord SD, Steel JL, et al. Fatigue in patients receiving maintenance dialysis: a review of definitions, measures, and contributing factors. American Journal of Kidney Diseases 2008;52:353-65.

14. Manns B, Hemmelgarn B, Lillie E, et al. Setting research priorities for patients on or Nearing dialysis. CJASN 2014;9:1813-21.

15. Johansen KL, Finkelstein FO, Revicki DA, et al. Systematic review of the impact of erythropoiesis-stimulating agents on fatigue in dialysis patients. Nephrology Dialysis Transplantation 2012;27:2418-25.

16. Song Y-yuan, Hu R-jun, Diao Y-shu, Song Y, Hu R, Diao Y, et al. Effects of exercise training on restless legs syndrome, depression, sleep quality, and fatigue among hemodialysis patients: a systematic review and meta-analysis. J Pain Symptom Manage 2018;55:1184-95.

17. Delgado C, Johansen KL. Barriers to exercise participation among dialysis patients. Nephrology Dialysis Transplantation 2012;27:1152-7.

18. van den Akker LE, Beckerman $\mathrm{H}$, Collette $\mathrm{EH}$, et al. Effectiveness of cognitive behavioral therapy for the treatment of fatigue in patients with multiple sclerosis: a systematic review and meta-analysis. J Psychosom Res 2016;90:33-42.

19. Kangas M, Bovbjerg DH, Montgomery GH. Cancer-Related fatigue: a systematic and meta-analytic review of non-pharmacological therapies for cancer patients. Psychol Bull 2008;134:700-41.

20. Mathiowetz VG, Finlayson ML, Matuska KM, et al. Randomized controlled trial of an energy conservation course for persons with multiple sclerosis. Mult Scler 2005;11:592-601.

21. Finlayson $\mathrm{M}$, Preissner $\mathrm{K}$, Cho $\mathrm{C}$, et al. Randomized trial of a teleconference-delivered fatigue management program for people with multiple sclerosis. Mult Scler 2011;17:1130-40.

22. Vanage SM, Gilbertson KK, Mathiowetz V. Effects of an energy conservation course on fatigue impact for persons with progressive multiple sclerosis. Am J Occup Ther 2003;57:315-23.

23. Kim YJ, Rogers JC, Raina KD, et al. Solving fatigue-related problems with cardiac arrest survivors living in the community. Resuscitation 2017;118:70-4.

24. Norberg E-B, Löfgren B, Boman K, et al. A client-centred programme focusing energy conservation for people with heart failure. Scand J Occup Ther 2017;24:455-67.

25. Farragher JF. Developing "PEP": A Personalized, Web-Supported Energy Conservation Education Program for People on Chronic Dialysis Therapy with Fatigue [Internet]. [Toronto]: University of Toronto; 2018 [cited 2019 Apr 23]. Available from:. Available: https:// tspace.library.utoronto.ca/handle/1807/91865

26. Missiuna C, Mandich AD, Polatajko HJ, et al. Cognitive orientation to daily occupational performance (CO-OP). Phys Occup Ther Pediatr 2001;20:69-81.

27. Cameron D, Craig T, Edwards B, et al. Cognitive orientation to daily occupational performance (CO-OP): a new approach for children with cerebral palsy. Phys Occup Ther Pediatr 2017;37:183-98.

28. Medical Education Institute. Kidney School [Internet]. Kidney Schoolcited 2019 Mar 1]. Available: www.kidneyschool.org

29. Krupp LB, LaRocca NG, Muir-Nash J, et al. The fatigue severity scale: application to patients with multiple sclerosis and systemic lupus erythematosus. Arch Neurol 1989;46:1121-3.

30. Flachenecker P, Kümpfel T, Kallmann B, et al. Fatigue in multiple sclerosis: a comparison of different rating scales and correlation to clinical parameters. Mult Scler 2002;8:523-6.

31. Learmonth YC, Dlugonski D, Pilutti LA, et al. Psychometric properties of the fatigue severity scale and the modified fatigue impact scale. $J$ Neurol Sci 2013;331:102-7.

32. Fisk JD, Ritvo PG, Ross L, et al. Measuring the functional impact of fatigue: initial validation of the fatigue impact scale. Clin Infect Dis 1994;18(Supplement 1):S79-S83.

33. Wood-Dauphinee SL, Opzoomer MA, Williams Jl, et al. Assessment of global function: the reintegration to normal living index. Arch Phys Med Rehabil 1988;69:583-90. 
34. Bourget N, Deblock-Bellamy A, Blanchette AK, et al. Use and psychometric properties of the reintegration to normal living index in rehabilitation: a systematic review. Ann Phys Rehabil Med 2018;61:262-9.

35. Law M, Baptiste S, McColl M, et al. The Canadian occupational performance measure: an outcome measure for occupational therapy. Can J Occup Ther 1990;57:82-7.

36. Carswell A, McColl MA, Baptiste S, et al. The Canadian occupationa performance measure: a research and clinical literature review. Can $J$ Occup Ther 2004:71:210-22.

37. Whitehead AL, Julious SA, Cooper CL, et al. Estimating the sample size for a pilot randomised trial to minimise the overall trial sample size for the external pilot and main trial for a continuous outcome variable. Stat Methods Med Res 2016;25:1057-73.
38. Van Heest KNL, Mogush AR, Mathiowetz VG. Effects of a one-to-one fatigue management course for people with chronic conditions and fatigue. Am J Occup Ther 2017;71.

39. Holberg C, Finlayson M. Factors influencing the use of energy conservation strategies by persons with multiple sclerosis. Am J Occup Ther 2007;61:96-107.

40. Raina KD, Morse JQ, Chisholm D, et al. Feasibility of a cognitive behavioral intervention to manage fatigue in individuals with traumatic brain injury. Journal of Head Trauma Rehabilitation 2016;31:E41-E49.

41. Young GR, Conservation E. Occupational therapy, and the treatment of post-polio sequelae. Orthopedics 1991;14:1233-9. 